International Mathematical Forum, 2, 2007, no. 45, 2207 - 2220

\title{
Noether Symmetries and Conserved Momenta of Dirac Equation in Presymplectic Dynamics
}

\author{
Renato Grassini \\ Department of Mathematics and Applications \\ University of Naples Federico II \\ Via Cintia, Monte S. Angelo - 80126 Naples, Italy \\ renato.grassini@dma.unina.it
}

\begin{abstract}
Presymplectic dynamics, as it arises from the Lagrangian and Hamiltonian dynamics of 'non-regular' mechanical systems, has proved to be a theory whose focus is an implicit differential equation called Dirac equation [3]. A general geometric framework developped for implicit differential equations [4] will be applied to Dirac equation, with the aim of gaining a clear understanding of its possible symmetries and their role in the problem of integration. Noether's theory of symmetries and conserved momenta will then be extended to Dirac equation, and thence to non-regular Lagrangian and Hamiltonian dynamics. The overall picture will appear to be the most natural generalization of the classical theory concerning 'regular' mechanical systems.
\end{abstract}

\section{Mathematics Subject Classification: $70 \mathrm{H} 45$}

Keywords: presymplectic dynamics, implicit differential equations, symmetries, momentum mappings

\section{Introduction}

Symmetries and conservation laws are well established concepts for a system of ordinary differential equations in normal form, described - in geometric terms - as a vector field on a manifold.

Particularly rich is their history in symplectic dynamics (arising from the Lagrangian and Hamiltonian dynamics of 'regular' mechanical systems), where the law of the motion is, in coordinate formalism, a system of ordinary Hamiltonian equations and, in geometric formalism, a Hamiltonian vector field on 
a symplectic manifold. In such a case, Noether-type symmetries and their momentum mappings have found a firm and elegant geometric formulation [1].

Somewhat more nebulous is the situation in presymplectic dynamics (arising from the Lagrangian and Hamiltonian dynamics of 'non-regular' mechanical systems in Dirac's sense [7]), where the law of the motion is, in coordinate formalism, a system of ordinary differential equations in implicit form. In that case, the very geometrical formulation of the above law -usually conceived as a family of vector fields on a presymplectic manifold $[8,9,5,10]$ - appears unrelated to the original conceptions of classical Analysis and Mechanics and actually obscures the ultimate geometric meaning of 'implicit differential equation'.

The proper geometric definition of such an object, as has been recognized elsewhere [11,12], is simply that of a submanifold $\mathcal{D}$ of the tangent bundle of the given manifold $M$ (the image of a vector field $X$ on $M$ is the special case of a normal equation). Solutions to $\mathcal{D}$ will then be the smooth curves of $M$ whose tangent lifts live in $\mathcal{D}$ (in particular, the integral curves of $X$ in the normal case).

Once based on that conception, presymplectic dynamics appears to be the theory of 'Dirac equation' (a special kind of implicit differential equation on a presymplectic manifold), spontaneous generalization of 'Hamilton equation' (image of a Hamiltonian vector field on a symplectic manifold), and has been shown to be the 'common core' of both Lagrangian and Hamiltonian dynamics $[3]$.

In the present paper, Dirac equation will be cast into the general framework developped in [4] for implicit differential equations on manifolds (sec.2). That will lead to a clear understanding of the whole hierarchy of symmetries of Dirac equation and their role in the problem of integration (sec.3). In this context, Noether-type symmetries and their momentum mappings will find a natural generalization to Dirac equation (sec.4). Through this way, classical Noetherian conservation laws will be extended to both Lagrangian and Hamiltonian dynamics of non-regular mechanical systems (sec.5 and sec.6).

\section{Dirac equation in presymplectic dynamics}

We preliminarly recall the main features of presymplectic dynamics, as it arises from Lagrangian and Hamiltonian dynamics [3].

Let $M$ be a smooth manifold and

$$
\tau_{M}: T M \rightarrow M, \pi_{M}: T^{*} M \rightarrow M
$$

the tangent and the cotangent bundle of $M$, respectively. 
If

$$
\omega: M \rightarrow T^{*} M \otimes T^{*} M: p \mapsto \omega(p)
$$

is a presymplectic structure (i.e. a closed 2-form) on $M$ with 'musical' vector bundle morphism

$$
b: T M \rightarrow T^{*} M: x \in T_{p} M \mapsto{ }^{b} x:=i_{x} \omega:=\langle\omega(p) \mid x, \cdot\rangle \in T_{p}^{*} M
$$

(where the brackets denote pairing between forms and vectors), then

$$
\text { Graph } b:=\left\{(x, \theta) \subset T M \times\left._{M} T^{*} M\right|^{b} x=\theta\right\}
$$

is a Dirac structure on $M[6]$.

In analytical dynamics, where $M$ is the (velocity or momentum) phase space of a mechanical system and $T^{*} M$ is the space of covector forces of the system, a Dirac structure like Graph $b$ is assumed as a sort of balance law, prescribing condition

$$
\operatorname{Im}(\dot{\kappa}, \vartheta) \subset \text { Graph } b
$$

i.e.

$$
{ }^{b} \dot{\kappa}=\vartheta
$$

to each possible time-evolution

$$
(\kappa, \vartheta): I \subset \mathbb{R} \rightarrow M \times T^{*} M
$$

of the system (above $\dot{\kappa}$ denotes the tangent lift of $\kappa$ ).

In particular, by virtue of Graph b, to any covector force field (closed 1-form)

$$
\eta: M \rightarrow T^{*} M: p \mapsto \eta(p)
$$

there corresponds on $M$ the implicit differential equation

$$
\begin{aligned}
\mathcal{D}: & =\left\{x \in T M \mid\left(x, \eta\left(\tau_{M}(x)\right)\right) \in \text { Graph } b\right\} \\
& =\left\{\left.x \in T M\right|^{b} x=\eta\left(\tau_{M}(x)\right)\right\}
\end{aligned}
$$

called Dirac equation generated by $(\omega, \eta)$ (clearly, if $\omega$ is symplectic -i.e. b is invertible- and $\eta$ is exact -say $\eta=d H$ - then we obtain $\mathcal{D}=\operatorname{Im}{ }^{\sharp} d H$ with $\sharp:=b^{-1}$, i.e. $\mathcal{D}$ is the typical Hamilton equation of symplectic dynamics).

The solutions or integral curves of $\mathcal{D}$ are the smooth curves $\kappa$ 's of $M$ satisfying

$$
\operatorname{Im} \dot{\kappa} \subset \mathcal{D}
$$

i.e.

$$
{ }^{b} \dot{\kappa}=\eta \circ \kappa
$$


which is then to be regarded as a necessary condition for a motion $\kappa$ to be dynamically possible under the given force field $\eta$.

In both Lagrangian dynamics (on velocity phase space) and Hamiltonian dynamics (on momentum phase space), a Dirac equation may not be sufficient to characterize the dynamically possible motions, which in fact correspond to the integral curves of a more restrictive equation, extracted from one like $\mathcal{D}$ through an additional 'second-order' condition (see sec.5 and sec.6).

In any case, the dynamically possible motions are to be searched for among the integral curves of a Dirac equation, whose integration is therefore the first relevant step in dynamics.

\section{$3 \quad$ Noether symmetries}

Symmetries of an implicit differential equation on a manifold, are local or global transformations of the manifold which take integral curves onto integral curves [4]. Their primary role in the problem of integration is of course that of constructing new integral curves from known ones.

Here the symmetries of Dirac equation will be classified.

Let $\mathcal{D}$ be the Dirac equation generated on $M$ by $(\omega, \eta)$.

For any two open subsets $U$ and $V$ of $M$, a diffeomorphism

$$
\Phi: U \rightarrow V
$$

is a (local) dynamical symmetry of $\mathcal{D}$ if both $\Phi$ and $\Phi^{-1}$ take integral curves onto integral curves, i.e.

$$
\begin{aligned}
& \operatorname{Im} \kappa \subset U, \operatorname{Im} \dot{\kappa} \subset \mathcal{D} \Longrightarrow \operatorname{Im}(\Phi \circ \kappa) \subset \mathcal{D} \\
& \operatorname{Im} \kappa \subset V, \operatorname{Im} \dot{\kappa} \subset \mathcal{D} \Longrightarrow \operatorname{Im}\left(\Phi^{-1} \circ \kappa\right) \subset \mathcal{D}
\end{aligned}
$$

(a symmetry is said to be global, if $U=V=M$ ). Owing to the identities

$$
(\Phi \circ \kappa)^{\cdot}=\Phi_{*} \circ \dot{\kappa},\left(\Phi^{-1} \circ \kappa\right)^{\cdot}=\Phi_{*}^{-1} \circ \dot{\kappa}
$$

(where $\Phi_{*}$ denotes the tangent mapping of $\Phi$ and $\Phi_{*}^{-1}$ coincides with the tangent mapping of $\Phi^{-1}$ ), the above definition amounts to requiring that the integrable part $\mathcal{D}^{(\mathrm{i})}$ of $\mathcal{D}$-i.e. the region of $\mathcal{D}$ swept by the tangent lifts of its own integral curves- should be $\Phi$-invariant, which means

$$
\Phi_{*}\left(\mathcal{D}^{(\mathrm{i})} \cap T U\right)=\mathcal{D}^{(\mathrm{i})} \cap T V
$$

Thence one is naturally led to focus on a more 'formal' kind of symmetry, namely a geometrical symmetry $\Phi$ of $\mathcal{D}$, defined by requiring that $\mathcal{D}$ itself should be $\Phi$-invariant, i.e.

$$
\Phi_{*}(\mathcal{D} \cap T U)=\mathcal{D} \cap T V
$$


It is quite obvious, in view of the above mentioned identities, that a geometrical symmetry is a dynamical symmetry as well.

Finally one might even go back to the very generators $(\omega, \eta)$ of $\mathcal{D}$ and define a Noetherian symmetry $\Phi$ of $\mathcal{D}$ by requiring that $\omega$ and $\eta$ should be $\Phi$-invariant, that is to say,

$$
\Phi^{*}\left(\left.\omega\right|_{V}\right)=\left.\omega\right|_{U}, \Phi^{*}\left(\left.\eta\right|_{V}\right)=\left.\eta\right|_{U}
$$

(a restriction like $\left.\omega\right|_{V}$ is the pull back of $\omega$ by inclusion $V \hookrightarrow M$ and a symbol like $\Phi^{*}\left(\left.\omega\right|_{V}\right)$ denotes the pull back of $\left.\omega\right|_{V}$ by $\left.\Phi\right)$.

Such new symmetries still fall within the range of dynamical symmetries, as will now be shown.

Theorem 1 A Noetherian symmetry is a geometrical-and hence a dynamicalsymmetry.

Proof. It will suffice to prove that, for any diffeomorphism $\Phi: U \rightarrow V$,

$$
\Phi^{*}\left(\left.\omega\right|_{V}\right)=\left.\omega\right|_{U}, \Phi^{*}\left(\left.\eta\right|_{V}\right)=\left.\eta\right|_{U} \Longrightarrow \Phi_{*}(\mathcal{D} \cap T U) \subset \mathcal{D}
$$

To that end, for any $p \in U$ and $x \in T_{p} M$, put $q:=\Phi(p) \in V$ and $y:=\Phi_{* p}(x) \in$ $T_{q} M$ (with $\Phi_{* p}:=\left.\Phi_{*}\right|_{T_{p} M}$ ). From our hypotheses, it follows that

$$
{ }^{b} x=\langle\omega(p) \mid x, \cdot\rangle=\left\langle\omega(q) \mid \Phi_{* p}(x), \Phi_{* p}(\cdot)\right\rangle=\left\langle\omega(q) \mid y, \Phi_{* p}(\cdot)\right\rangle={ }^{b} y \circ \Phi_{* p}
$$

and

$$
\eta(p)=\eta(q) \circ \Phi_{* p}
$$

So, if $x \in \mathcal{D}$, i.e.

$$
{ }^{\mathrm{b}} x=\eta(p)
$$

one has

$$
{ }^{b} y \circ \Phi_{* p}=\eta(q) \circ \Phi_{* p}
$$

hence $\left(\Phi_{* p}\right.$ being an isomorphism)

$$
{ }^{b} y=\eta(q)
$$

i.e. $y \in \mathcal{D}$. That proves our claim.

To the above hierarchy of 'finite' symmetries, there corresponds a parallel hierarchy of 'infinitesimal' ones.

Let $X \in \chi(M)$ be a vector field on $M$, i.e. the infinitesimal generator of a one-parameter (local or global) group of transformations $\left\{\Phi_{t}: U_{t} \rightarrow V_{t}\right\}_{t \in \mathbb{R}}$. $X$ will be called a dynamical, geometrical or Noetherian infinitesimal symmetry 
of $\mathcal{D}$, if each diffeomorphism $\Phi_{t}$ is a (local or global) symmetry of $\mathcal{D}$ of the respective type.

More generally, let $g$ be a Lie algebra acting on $M$ through a Lie algebra homomorphism

$$
g \rightarrow \chi(M): \zeta \mapsto \zeta_{M}
$$

$g$ will be called a dynamical, geometrical or Noetherian symmetry algebra of $\mathcal{D}$, if each vector field $\zeta_{M}$ is an infinitesimal symmetry of $\mathcal{D}$ of the respective type (in such a case, if each $\zeta_{M}$ is complete, any Lie group with Lie algebra $g$ will act on $M$-through an action generated by the $\zeta_{M}$ 's- as a group of global symmetries of $\mathcal{D}$ ).

In the sequel, we shall deal with a special kind of Noetherian symmetry algebra $g$ of $\mathcal{D}$. To this purpose, recall that, for any $\zeta \in g$, the requirement of $\zeta_{M}$ being a Noetherian infinitesimal symmetry can as well be expressed by conditions

$$
d_{\zeta_{M}} \omega=0, d_{\zeta_{M}} \eta=0
$$

(where $d_{\zeta_{M}}=d i_{\zeta_{M}}+i_{\zeta_{M}} d$ is the Lie derivative along a vector field $\zeta_{M}$, expressed in terms of exterior derivative $d$ and interior product $i_{\zeta_{M}}$ ). As $\omega$ and $\eta$ are closed $(d \omega=0, d \eta=0)$, such conditions take the form

$$
d i_{\zeta_{M}} \omega=0, d i_{\zeta_{M}} \eta=0
$$

owing to which ${ }^{b} \zeta_{M}=i_{\zeta_{M}} \omega$ is required to be locally exact and $i_{\zeta_{M}} \eta$ is required to be locally constant.

By strengthening these requirements, we can prolong further our hierarchy of symmetry algebras.

Namely, we shall consider an exact Noetherian symmetry algebra of $\mathcal{D}$, i.e. a Lie algebra $g$ acting on $M$ in such a way that, for each $\zeta \in g, \zeta_{M}$ is taken by b onto a globally exact 1 -form

$$
{ }^{b} \zeta_{M}=d J_{\zeta_{M}}
$$

and is annihilated by $\eta$

$$
i_{\zeta_{M}} \eta=0
$$

Properties and examples of exact Noetherian symmetry algebras will be shown in the next sections.

\section{Momentum mappings}

The geometric formulation of Noether's theory of symmetries and corresponding conserved momenta -well established for Hamilton equation- can naturally be extended to Dirac equation, as will now be shown. 
We shall consider an exact Noetherian symmetry algebra $g$ of Dirac equation $\mathcal{D}$ and its dual space $g^{*}$ (a multiplicative notation will denote pairing between elements of $g^{*}$ and $g$ ).

Theorem 2 An exact Noetherian symmetry algebra $g$ of $\mathcal{D}$ admits a momentum mapping, i.e. a smooth mapping

$$
J: M \rightarrow g^{*}
$$

such that, for all $\zeta \in g$,

$$
{ }^{b} \zeta_{M}=d(J \zeta)
$$

where

$$
J \zeta: M \rightarrow \mathbb{R}: p \mapsto J(p) \zeta
$$

is the pairing between $J$ and $\zeta$.

Proof. Let $\left(\epsilon_{\alpha}\right)$, with $\alpha=1, \ldots, \operatorname{dim} g$, be a basis of $g$ and $\left(\epsilon^{\alpha}\right)$ the dual basis in $g^{*}$. For each value of $\alpha,{ }^{b} \epsilon_{\alpha M}$ is an exact 1 -form, i.e.

$$
{ }^{b} \epsilon_{\alpha M}=d J_{\alpha}
$$

for some smooth, real-valued function

$$
J_{\alpha}: M \rightarrow \mathbb{R}
$$

Put

$$
J:=J_{\alpha} \epsilon^{\alpha}: M \rightarrow g^{*}: p \mapsto J(p):=J_{\alpha}(p) \epsilon^{\alpha}
$$

(summation over $\alpha$ is understood). For any $\zeta=\zeta^{\alpha} \epsilon_{\alpha} \in g$, we obtain (by linearity)

$$
\begin{aligned}
{ }^{b} \zeta_{M} & ={ }^{b}\left(\zeta^{\alpha} \epsilon_{\alpha}\right)_{M}={ }^{b}\left(\zeta^{\alpha} \epsilon_{\alpha M}\right)=\zeta^{\alpha}\left({ }^{b} \epsilon_{\alpha M}\right)=\zeta^{\alpha}\left(d J_{\alpha}\right) \\
& =d\left(J_{\alpha} \zeta^{\alpha}\right)=d\left(J_{\alpha}\left(\epsilon^{\alpha} \zeta\right)\right)=d\left(\left(J_{\alpha} \epsilon^{\alpha}\right) \zeta\right) \\
& =d(J \zeta)
\end{aligned}
$$

which is our claim.

Theorem 3 The momentum mapping $J$ of an exact Noetherian symmetry algebra $g$ of $\mathcal{D}$ is a constant of the motion of $\mathcal{D}$, i.e. J satisfies the conservation law

$$
J \circ \kappa=\text { const. }
$$

along each integral curve $\kappa$ of $\mathcal{D}$. 
Proof. For any $\zeta \in g$, let

$$
(J \zeta)^{T}: T M \rightarrow \mathbb{R}
$$

be the tangent lift of $J \zeta$, defined by putting -for all $p \in M$ -

$$
\left.(J \zeta)^{T}\right|_{T_{p} M}:=d_{p}(J \zeta):=(J \zeta)_{* p}: T_{p} M \rightarrow T_{(J \zeta)(p)} \mathbb{R}=\mathbb{R}
$$

On the one hand, owing to Leibniz rule, $(J \zeta)^{T}$ is expressed by

$$
(J \zeta)^{T}=J^{T} \zeta
$$

where

$$
J^{T}: T M \rightarrow g^{*}
$$

is the tangent lift of $J$, defined by putting -for all $p \in M$ -

$$
\left.J^{T}\right|_{T_{p} M}:=d_{p} J:=J_{* p}: T_{p} M \rightarrow T_{J(p)} g^{*}=g^{*}
$$

and

$$
J^{T} \zeta: T M \rightarrow \mathbb{R}: x \mapsto J^{T}(x) \zeta
$$

is the pairing between $J^{T}$ and $\zeta$.

On the other hand, $(J \zeta)^{T}$ is expressed on $\mathcal{D}$ by

$$
\left.(J \zeta)^{T}\right|_{\mathcal{D}}=-\left.i_{\zeta_{M}} \eta \circ \tau_{M}\right|_{\mathcal{D}}
$$

since, if $x \in \mathcal{D}$ (i.e. ${ }^{b} x=\eta(p)$, with $p:=\tau_{M}(x)$ ), we have

$$
\begin{aligned}
(J \zeta)^{T}(x) & =\left\langle d_{p}(J \zeta) \mid x\right\rangle=\left\langle{ }^{b} \zeta_{M}(p) \mid x\right\rangle=-\left\langle{ }^{b} x \mid \zeta_{M}(p)\right\rangle \\
& =-\left\langle\eta(p) \mid \zeta_{M}(p)\right\rangle=-\left\langle\eta \mid \zeta_{M}\right\rangle(p) \\
& =-\left(i_{\zeta_{M}} \eta \circ \tau_{M}\right)(x)
\end{aligned}
$$

As $\zeta_{M}$ is annihilated by $\eta$, i.e. $i_{\zeta_{M}} \eta=0$, we obtain

$$
\left.\left(J^{T} \zeta\right)\right|_{\mathcal{D}}=\left.(J \zeta)^{T}\right|_{\mathcal{D}}=0
$$

So it has been established that

$$
J^{T}(x) \zeta=0, \forall x \in \mathcal{D}, \forall \zeta \in g
$$

which means

$$
J^{T}(x)=0, \forall x \in \mathcal{D}
$$

that is to say,

$$
\left.J^{T}\right|_{\mathcal{D}}=0
$$


Hence

$$
J \circ \kappa)^{\cdot}=J^{T} \circ \dot{\kappa}=0
$$

whenever $\operatorname{Im} \dot{\kappa} \subset \mathcal{D}$, which proves our claim.

As a consequence, the role of an exact Noetherian symmetry algebra $g$ in the problem of integrating $\mathcal{D}$ (under suitable hypotheses on the rank of $J$ ) is that of 'reducing the number of degrees of freedom'. Indeed, in view of the above conservation law, any Cauchy problem $\left(M, \mathcal{D}, x_{\circ}, t_{\circ}\right)$-expressed by $\operatorname{Im} \dot{\kappa} \subset \mathcal{D}$ and $\dot{\kappa}\left(t_{\circ}\right)=x_{\circ}$, with $x_{\circ} \in \mathcal{D}^{(i)}$ and $t_{\circ} \in \mathbb{R}$ - will admit the same solution curves as $\left(M_{\mu}, \mathcal{D}_{\mu}, x_{\circ}, t_{\circ}\right)$, where $\mathcal{D}_{\mu}:=\mathcal{D} \cap T M_{\mu}$ is the 'restriction' of $\mathcal{D}$ to the lower-dimensional submanifold $M_{\mu}:=J^{-1}(\mu) \subset M$ with $\mu:=J\left(\tau_{M}\left(x_{\circ}\right)\right)$ $[4]$.

\section{Applications to Lagrangian dynamics}

The above results will now be applied to a simple kind of constrained mechanical system, described by the following ingredients:

(i) a smooth manifold $Q$ (the configuration space of the system), (ii) a real-valued smooth function $L$ (the Lagrangian of the system) defined on an open submanifold $M$ of $T Q$.

Lagrangian dynamics deals with the problem of characterizing, through a suitable law, the dynamically possible motions $\gamma$ 's of the system in configuration space $Q$ or, equivalently, the dynamically possible motions $\kappa$ 's of the system in velocity phase space $T Q$, which are obtained from -and are bijectively related to- the dynamically possible motions in $Q$ via tangent lifting $\gamma \mapsto \kappa:=\dot{\gamma}$

The dynamically possible motions in $T Q$, determined by the established law (Hamilton's variational principle of stationary action), have been shown [2] to be the integral curves of the implicit differential equation on $M \subset T Q$ defined as follows.

Let

$$
\theta:=d_{S} L=i_{S} d L, E:=d_{\Delta} L-L
$$

be the Poincaré-Cartan 1-form and the energy associated with the Lagrangian (where $\Delta$ denotes the dilation vector field on $M, S$ the vertical endomorphism of $T M, i_{S}$ the corresponding derivation of type $i_{*}$ and $\left.d_{S}:=i_{S} d-d i_{S}\right)$.

Put

$$
\omega:=-d \theta, \eta:=d E
$$

and consider the Dirac equation on $M$ generated by $(\omega, \eta)$, i.e.

$$
\mathcal{D}:=\left\{\left.x \in T M\right|^{b} x=\eta\left(\tau_{M}(x)\right)\right\}
$$


From $\mathcal{D}$ we extract a more restrictive equation by adding the second-order condition defining

$$
T^{2} Q:=\left\{x \in T T Q \mid \tau_{T Q}(x)=\tau_{Q *}(x)\right\}
$$

By doing so, we obtain the announced Euler-Lagrange equation

$$
\mathcal{E}:=\mathcal{D} \cap T^{2} Q
$$

whose integral curves $\kappa$ 's will be those of $\mathcal{D}$ satisfying the second-order condition $\kappa=\dot{\gamma}$, with $\gamma:=\tau_{Q} \circ \kappa$.

If $L$ is a regular Lagrangian (i.e. ${ }^{b}$ is invertible), then $\mathcal{E}=\mathcal{D} \subset T^{2} Q$ is a normal Hamilton equation, for which a well known Noether's theorem on 'point-symmetries' holds true. Owing to the general theory developped in sec.4, Noether's theorem can be extended to the implicit equations $\mathcal{D}$ and $\mathcal{E}$ corresponding to an arbitrary (possibly non-regular) Lagrangian, as follows.

Let $g$ be a Lie algebra acting on 'points' (i.e. on $Q$ ) through a Lie algebra homomorphism

$$
g \rightarrow \chi(Q): \zeta \mapsto \zeta_{Q}
$$

and hence on 'velocities', i.e. on $M \subset T Q$, through

$$
g \rightarrow \chi(M): \zeta \mapsto \zeta_{M}:=\left.\zeta_{Q}^{T}\right|_{M}
$$

(where $\zeta_{Q}^{T}$ denotes the tangent lift of $\zeta_{Q}$ ).

$L$ will be assumed to be $g$-invariant, i.e.

$$
d_{\zeta_{M}} L=0, \forall \zeta \in g
$$

Theorem 4 If $L$ is $g$-invariant, $g$ is an exact Noetherian symmetry algebra of $\mathcal{D}$ with momentum map $J: M \rightarrow g^{*}$ given by

$$
J \zeta:=d_{S \zeta_{M}} L, \forall \zeta \in g
$$

$J$ is then a constant of the motion of both $\mathcal{D}$ and $\mathcal{E}$.

Proof. We shall make use of some known properties of derivations associated with vector forms, namely

$i_{S} d_{\zeta_{M}}-d_{\zeta_{M}} i_{S}=i_{\left[S, \zeta_{M}\right]}, i_{\zeta_{M}} d_{S}+d_{S} i_{\zeta_{M}}=d_{S \zeta_{M}}-i_{\left[S, \zeta_{M}\right]}, d_{\zeta_{M}} d_{\Delta}-d_{\Delta} d_{\zeta_{M}}=d_{\left[\zeta_{M}, \Delta\right]}$

where $[\cdot, \cdot]$ is the Lie bracket of vector forms, and

$$
\left[S, \zeta_{M}\right]=0,\left[\zeta_{M}, \Delta\right]=0
$$


(since $\zeta_{M}$ is a tangent lift). Owing to such properties, for any $\zeta \in g$, we have

$$
\begin{aligned}
& { }^{b} \zeta_{M}=i_{\zeta_{M}} \omega=-i_{\zeta_{M}} d d_{S} L=d i_{\zeta_{M}} d_{S} L-d_{\zeta_{M}} i_{S} d L=d d_{S \zeta_{M}} L-i_{S} d d_{\zeta_{M}} L \\
& i_{\zeta_{M}} \eta=d_{\zeta_{M}} E=d_{\zeta_{M}}\left(d_{\Delta} L-L\right)=d_{\Delta} d_{\zeta_{M}} L-d_{\zeta_{M}} L
\end{aligned}
$$

whence, under hypothesis $d_{\zeta_{M}} L=0$,

$$
{ }^{b} \zeta_{M}=d(J \zeta), i_{\zeta_{M}} \eta=0
$$

That proves that $g$ is an exact Noetherian symmetry algebra of $\mathcal{D}$ with the claimed momentum mapping $J$.

Owing to the previous theorem, $J$ is a constant of the motion of $\mathcal{D}$ and hence of $\mathcal{E} \subset \mathcal{D}$.

\section{Applications to Hamiltonian dynamics}

The dynamics of the above mechanical system will now be reconsidered under the following almost-regularity hypotheses (which involve the Legendre morphism $\mathcal{L}: M \rightarrow T^{*} Q$, satisfying $\pi_{Q} \circ \mathcal{L}=\left.\tau_{Q}\right|_{M}$, defined by the fibre derivative of Lagrangian $L)$ :

(i) $M_{1}:=\operatorname{Im} \mathcal{L}$ is an embedded submanifold of $T^{*} Q$,

(ii) $\mathcal{L}_{1}: M \rightarrow M_{1}$ - defined by $\iota_{1} \circ \mathcal{L}_{1}=\mathcal{L}$ with $\iota_{1}: M_{1} \hookrightarrow T^{*} Q$ - is a submersion,

(iii) $E=\mathcal{L}_{1}^{*}\left(E_{1}\right)$, for some $E_{1}: M_{1} \rightarrow \mathrm{R}$.

Hamiltonian dynamics deals with the problem of characterizing the $d y$ namically possible motions $\kappa_{1}$ 's of the system in momentum phase space $T^{*} Q$, which are obtained from -and are bijectively related to- the dynamically possible motions $\gamma$ 's in $Q$ via Legendre lifting $\gamma \mapsto \kappa_{1}:=\mathcal{L} \circ \dot{\gamma}$. The dynamically possible motions in $T^{*} Q$ have been shown [3] to be the integral curves of the implicit differential equation on $M_{1} \subset T^{*} Q$ defined as follows.

Put

$$
\omega_{1}:=-d \theta_{1}, \eta_{1}:=d E_{1}
$$

(where $\theta_{1}$ denotes the pull back by $\iota_{1}$ of the Liouville 1 -form of $T^{*} Q$ ) and consider the Dirac equation on $M_{1}$ generated by $\left(\omega_{1}, \eta_{1}\right)$, i.e.

$$
\mathcal{D}_{1}:=\left\{\left.z \in T M_{1}\right|^{b_{1}} z=\eta_{1}\left(\tau_{M_{1}}(z)\right)\right\}
$$

From $\mathcal{D}_{1}$ we extract a more restrictive equation by adding the second-order condition defining

$$
T_{\mathcal{L}}^{2} Q:=\left\{z \in T^{*} T Q \mid \pi_{Q *}(z) \in M, \tau_{T^{*} Q}(z)=\mathcal{L}\left(\pi_{Q *}(z)\right)\right\}
$$


By doing so, we obtain the announced Hamilton-Dirac equation

$$
\mathcal{H}:=\mathcal{D}_{1} \cap T_{\mathcal{L}}^{2} Q
$$

whose integral curves $\kappa_{1}$ 's will be those of $\mathcal{D}_{1}$ which satisfy the second-order condition $\kappa_{1}=\mathcal{L} \circ \dot{\gamma}$, with $\gamma:=\pi_{Q} \circ \kappa_{1}$.

We shall now extend Noether's theorem on 'point symmetries' to the above implicit equations $\mathcal{D}_{1}$ and $\mathcal{H}$. To this purpose, we need a Lie algebra $g$ acting on $Q$, and hence on $M \subset T Q$, in such a way that, for each $\zeta \in g, \zeta_{M} \in \chi(M)$ satisfies the projectability condition

$$
\mathcal{L}_{1 *} \circ \zeta_{M}=\zeta_{M_{1}} \circ \mathcal{L}_{1}
$$

with $\zeta_{M_{1}} \in \chi\left(M_{1}\right)$, which allows the action of $g$ to be lifted to 'momenta' as well, i.e. to $M_{1} \subset T^{*} Q$, through

$$
g \rightarrow \chi\left(M_{1}\right): \zeta \mapsto \zeta_{M_{1}}
$$

Theorem 5 If $L$ is g-invariant, $g$ is a Noetherian symmetry algebra of $\mathcal{D}_{1}$ with momentum mapping $J_{1}: M_{1} \rightarrow g^{*}$ given by

$$
J_{1} \zeta:=\left\langle i d_{M_{1}}\left|\zeta_{Q} \circ \pi_{Q}\right|_{M_{1}}\right\rangle, \forall \zeta \in g
$$

$J_{1}$ is then a constant of the motion of both $\mathcal{D}_{1}$ and $\mathcal{H}$.

Proof. First, we reconsider the momentum mapping $J$ defined in sec.5, whose pairing with any $\zeta \in g$ gives

$$
J \zeta=\mathcal{L}_{1}^{*}\left(J_{1} \zeta\right)
$$

since

$$
\begin{aligned}
J \zeta & :=d_{S \zeta_{M}} L=\left\langle d L \mid S \zeta_{M}\right\rangle=\left\langle\mathcal{L}_{1} \mid \tau_{Q *} \circ \zeta_{M}\right\rangle=\left\langle\mathcal{L}_{1}\left|\zeta_{Q} \circ \tau_{Q}\right|_{M}\right\rangle=\left\langle\mathcal{L}_{1} \mid \zeta_{Q} \circ \pi_{Q} \circ \mathcal{L}_{1}\right\rangle \\
& =\left\langle i d_{M_{1}}\left|\zeta_{Q} \circ \pi_{Q}\right|_{M_{1}}\right\rangle \circ \mathcal{L}_{1}=J_{1} \zeta \circ \mathcal{L}_{1}
\end{aligned}
$$

Moreover, we recall that $\omega=\mathcal{L}_{1}^{*}\left(\omega_{1}\right)$, whence

$$
{ }^{b} \zeta_{M}=\mathcal{L}_{1}^{*}\left({ }^{b_{1}} \zeta_{M_{1}}\right)
$$

since (for all $p \in M$ )

$$
\begin{aligned}
{ }^{b} \zeta_{M}(p) & =\left\langle\omega(p) \mid \zeta_{M}(p), \cdot\right\rangle=\left\langle\omega_{1}\left(\mathcal{L}_{1}(p)\right) \mid \mathcal{L}_{1 * p}\left(\zeta_{M}(p)\right), \mathcal{L}_{1 * p}(\cdot)\right\rangle \\
& =\left\langle\omega_{1}\left(\mathcal{L}_{1}(p)\right) \mid \zeta_{M_{1}}\left(\mathcal{L}_{1}(p)\right), \mathcal{L}_{1 * p}(\cdot)\right\rangle={ }^{b} \zeta_{M_{1}}\left(\mathcal{L}_{1}(p)\right) \circ \mathcal{L}_{1 * p}
\end{aligned}
$$

Finally, we remark that $\eta=\mathcal{L}_{1}^{*}\left(\eta_{1}\right)$, whence

$$
i_{\zeta_{M}} \eta=\mathcal{L}_{1}^{*}\left(i_{\zeta_{M_{1}}} \eta_{1}\right)
$$


since

$$
\begin{aligned}
i_{\zeta_{M}} \eta & =\left\langle\eta \mid \zeta_{M}\right\rangle=\left\langle\eta_{1} \circ \mathcal{L}_{1} \mid \mathcal{L}_{1 *} \circ \zeta_{M}\right\rangle=\left\langle\eta_{1} \circ \mathcal{L}_{1} \mid \zeta_{M_{1}} \circ \mathcal{L}_{1}\right\rangle \\
& =\left\langle\eta_{1} \mid \zeta_{M_{1}}\right\rangle \circ \mathcal{L}_{1}=i_{\zeta_{M_{1}}} \eta_{1} \circ \mathcal{L}_{1}
\end{aligned}
$$

Now, as is known from sec.5, the hypothesis of $L$ being $g$-invariant implies

$$
{ }^{b} \zeta_{M}=d(J \zeta), i_{\zeta_{M}} \eta=0, \forall \zeta \in g
$$

which, owing to $(a),(b),(c)$ and recalling that $\mathcal{L}_{1}$ is a surjective submersion, read

$$
{ }^{b_{1}} \zeta_{M_{1}}=d\left(J_{1} \zeta\right), i_{\zeta_{M_{1}}} \eta_{1}=0, \forall \zeta \in g
$$

That proves that $g$ is a Noetherian symmetry algebra of $\mathcal{D}_{1}$ with the claimed momentum mapping $J_{1}$.

As a consequence, $J_{1}$ is a constant of the motion of $\mathcal{D}_{1}$ and hence of $\mathcal{H} \subset \mathcal{D}_{1}$.

\section{References}

[1] R. Abraham and J.E. Marsden, Foundations of Mechanics, $2^{\text {nd }}$ edn., Benjamin/Cummings (1978), Mass.

[2] F. Barone and R. Grassini, On the Second-Order Euler-Lagrange Equation in Implicit Form, Ric. Mat. 46 (1997), 221-233.

[3] F. Barone and R. Grassini, Generalized Hamiltonia Dynamics after Dirac and Tulczyjew, Banach Center Publ. 59 (2003).

[4] F. Barone and R. Grassini, Geometry of Implicit Differential Equations on Smooth Manifolds, Bull. Math.Soc. Sc. Math. Roumanie 47(95) No. 3-4. 2004,119-145.

[5] F. Cantrijn, J.F. Carinẽna, M. Crampin and L.A. Ibort, Reduction of Degenerate Lagrangians, J. Geom. Phys. 3 (1986), 353-400.

[6] T.J. Courant, Dirac Manifolds, Trans. Am. Math. Soc. 319 (1990), 631-661.

[7] P.A.M. Dirac, Generalized Hamiltonia Dynamics, Can. J. Math. (1950), 129-148. 
[8] M.J. Gotay, J.M. Nester and G. Hinds, Presymplectic Manifolds and the Dirac-Bergmann Theory of Constraints, J. Math. Phys. 19 (1978), 2388-2399.

[9] M.J. Gotay and J.M. Nester Presymplectic Lagrangian Systems I and II, Ann. Inst. Henri poincaré A 30 (1979), 129-142, and 32 (1980), 1-13.

[10] M. de León and D. Martin de Diego, Symmetries and Constants of the Motion for Singular Lagrangian Systems, Int. J. Theor. Phys. 35 (1996), 975-1011.

[11] P.J. Rabier and W.C. Rheinboldt, A Geometric Treatment of Implicit Differential-Algebraic Equations, J. Diff. Eqs 109 (1994),110-146.

[12] W.M. Tulczyjew, Geometric Formulations of Physical Theories, Bibliopolis (1989), Naples.

Received: February 9, 2007 